\title{
Iron and Nitrogen Co-doped Carbon Spheres as High Efficiency Oxygen Reduction Catalyst
}

\author{
Zhaoyan Chen ${ }^{1}$, Supeng Pei ${ }^{1,}$, Yueyang Sun ${ }^{1}$, Xia Xiong ${ }^{l}$, Wei Zhang ${ }^{1}$, Zhiyue Han ${ }^{l}$, Kangwei Xu ${ }^{l}$, \\ Zongshang Zhou ${ }^{1}$, Yongming Zhang ${ }^{2,3}$, Wei Feng ${ }^{3}$, Li Wang ${ }^{3}$, YeCheng Zou \\ ${ }^{1}$ School of Chemical and Environmental Engineering, Shanghai Institute of Technology, Shanghai \\ 201418, China. \\ ${ }^{2}$ School of Chemistry and Chemical Engineering, Center of Hydrogen Science, Shanghai Jiao Tong \\ University, Shanghai 200240, China. \\ ${ }^{3}$ State Key Laboratory of Fluorinated Functional Membrane Materials, Dongyue Group, Zibo 256401, \\ China. \\ *E-mail: peisupeng@126.com
}

doi: $10.20964 / 2021.05 .36$

Received: 7 November 2020 / Accepted: 16 December 2020 / Published: 31 March 2021

\begin{abstract}
Iron and nitrogen co-doped carbon spheres (Fe-N/CS) catalysts were prepared from iron nitrate nonahydrate by electrospinning and thermal treatment. The effect of different iron contents on the morphology and performance of the catalyst was explored by changing the amount of iron precursor. And the relationship between its structure and the oxygen reduction activity was also discussed. The results showed that there is an optimal content for the iron. When the iron content was $0.18 \%$ (Fe-N/CS0.18 ), the initial and half-wave potential of the catalyst were $0.970 \mathrm{~V}$ and $0.842 \mathrm{~V}$, which are the best result among the $\mathrm{Fe}-\mathrm{N} / \mathrm{CS}$ catalysts. Compared with that for commercial Pt/C, the Fe-N/CS-0.18 even showed a better ORR catalytic performance. The current retention of the Fe-N/CS-0.18 was $90.6 \%$ after continuous operation for $10000 \mathrm{~s}$, which is better than that for commercial Pt/C (84.2\%). Additionally, the methanol tolerance of Fe-N/CS-0.18 is better than that of the commercial Pt/C. The current density percentage of Fe-N/CS-0.18 has slightly decrease after the addition of methanol at $500 \mathrm{~s}$. However, the commercial $\mathrm{Pt} / \mathrm{C}$ decreases sharply. This was attributed to the abundant active nitrogen (pyridine nitrogen and pyrrole nitrogen) and suitable iron content in Fe-N/CS-0.18, which easily promote the oxygen reduction reaction.
\end{abstract}

Keywords: Iron-nitrogen codoped; Carbon sphere; Oxygen reduction catalyst

$\underline{\text { FULL TEXT }}$ 
(C) 2021 The Authors. Published by ESG (www.electrochemsci.org). This article is an open access article distributed under the terms and conditions of the Creative Commons Attribution license (http://creativecommons.org/licenses/by/4.0/). 